Schmerz 2010 $24: 559-560$

DOI 10.1007/s00482-010-0993-0

Online publiziert: 6 . November 2010

(c) Deutsche Gesellschaft zum Studium

des Schmerzes. Published by Springer-Verlag -

all rights reserved 2010

\author{
H.-G. Schaible \\ Physiologie I, Institut für Physiologie der Universität Jena
}

\section{Der schmale Grat zwischen Innovation und Risiko}

Unter den chronischen Schmerzen gehören Arthroseschmerzen zu den häufigsten Schmerzen. In einer großen europaweiten Studie zur Häufigkeit chronischer Schmerzen mit über 44.000 befragten Personen wurden Gelenkschmerzen und hierbei insbesondere Arthroseschmerzen am häufigsten genannt [3]. Da die meisten Arthrosen derzeit nicht kausal behandelt werden können, kommt der Schmerztherapie besondere Bedeutung zu. Zur Behandlung der Arthroseschmerzen werden v. a. nichtsteroidale Antirheumatika (NSAR) eingesetzt, und bei entsprechenden Voraussetzungen werden auch Opioide verordnet [1]. Insgesamt können diese Therapeutika die Schmerzproblematik bei Arthrose nicht befriedigend lösen, weshalb besonders auch für die Behandlung von Arthroseschmerzen Alternativen gesucht werden.

\section{Klinische Studien zur Neutralisierung von NGF}

Auf der Tagung der Deutschen Gesellschaft für Rheumatologie im September 2010 in Hamburg fand ein Symposium zum Stand der Arthroseforschung und der Schmerztherapie bei Arthrose statt. Im Rahmen dieses Symposiums hat der Verfasser dieses Editorials die Aufgabe bekommen, über neue Entwicklungen der Schmerztherapie bei Arthrose zu referieren. Insbesondere sollte im Fokus der neue Therapieansatz mit Antikörpern gegen „nerve growth factor“ (NGF) stehen. Nach Pressemitteilungen konnte die Firma Pfizer auf internationalen rheumatologischen Fachtagungen [z. B. auf dem
11. EULAR (European League Against Rheumatism)-Kongress 2010] über groß angelegte Phase-III-Studien berichten, in denen eine Schmerztherapie mit Tanezumab, einem monoklonalen Antikörper gegen NGF, insbesondere bei Arthroseschmerz sehr erfolgreich ist. Das Besondere an dieser neuen Therapieform ist, dass hier erstmals ein Antikörper speziell zum Zweck der Schmerztherapie eingesetzt wird. NGF wird als ein Schlüsselmolekül der Nozizeption betrachtet, weshalb die Neutralisierung von NGF sozusagen in das Zentrum der Nozizeption vorstößt (s. unten). Tatsächlich laufen bei mehreren großen Pharmafirmen Programme mit dem Ziel, Schmerzen durch die Neutralisierung von NGF zu bekämpfen. Während der Vorbereitung des Referates erschienen allerdings weitere Pressemitteilungen, in denen berichtet wurde, dass die laufenden klinischen Studien von Pfizer einstweilen angehalten wurden. Grund dafür war offensichtlich, dass bei einigen Patienten im Rahmen der Therapie eine rasante Verschlechterung der Arthrose auftrat (als Folge fehlender Schonung wegen der Schmerzreduktion?), sodass ein Gelenkersatz erforderlich wurde. Dies erfordert eine Abklärung, ob ein kausaler Zusammenhang zwischen der Therapie und der Verschlechterung der Krankheit besteht.

Der Versuch, durch Neutralisierung von NGF Schmerzen zu bekämpfen, ist aus mehreren Gründen wissenschaftlich und klinisch sehr interessant. Zum einen ist es eine spannende Frage, ob in absehbarer Zeit neue Behandlungsmöglichkeiten gegen Arthroseschmerzen und möglicher- weise andere chronische Schmerzen zur Verfügung stehen werden. Zum andern stellt sich die interessante Frage, wie weit man zum Zweck der Schmerztherapie in ein Mediatorsystem eingreifen kann, das zentral für die Biologie der Nozizeptoren ist. Schließlich ist von Interesse, ob „Antiköper gegen Schmerz“ eine Therapieoption der Zukunft sind. Dass man prinzipiell mit Antikörpern sehr gute therapeutische Erfolge erzielen kann, ist z. B. aus der Rheumatologie gut bekannt. Die Neutralisierung von Tumornekrosefaktor (TNF) durch Antikörper hat bei vielen Patienten die gelenkzerstörende Progression der rheumatoiden Arthritis aufgehalten.

\section{Wie wirkt NGF?}

NGF ist ein essenzieller Faktor für die normale Entwicklung des sympathischen Nervensystems und der Nozizeptoren. NGF wirkt über den trkA-Rezeptor. Eine Mutation dieses Rezeptors kann zu einer kongenitalen Schmerzunempfindlichkeit führen. Postnatal wird der trkA-Rezeptor in vielen Nozizeptoren herunterreguliert. Es bleibt aber ein großer Teil der CFasern weiterhin abhängig von NGF, während die übrigen C-Fasern vom Wachstumsfaktor GDNF abhängig werden. Interessanterweise scheinen nach einigen immunhistochemischen Untersuchungen die meisten Nozizeptoren der Gelenke und des Knochens im Gegensatz zu den kutanen Nozizeptoren von NGF abhängig zu bleiben. Abhängig von NGF heißt, dass für die Aufrechterhaltung des Phänotyps der trkA-exprimierenden Fasern NGF erforderlich ist. Entzieht man im 
Experiment dem Körper NGF, so nimmt die Empfindlichkeit dieser Nozizeptoren für thermische und chemische Reize $a b$, und Nervenfasern ziehen sich aus NGFfreien Strukturen zurück. Seit Langem ist bekannt, dass NGF in einem entzündeten Organ in erhöhtem Maße freigesetzt wird, und zwar aus verschiedenen nichtneuronalen Zellen. Zahlreiche Zytokine bewirken eine Hochregulation der mRNA von NGF, während Glukokortikoide und Interferone die gegenteilige Wirkung entfalten. NGF kann im Gewebe eine lang anhaltende mechanische und thermische Hyperalgesie erzeugen. Andererseits können Antikörper gegen NGF oder an trkA-Rezeptoren gekoppelte Fusionsproteine die thermische und - in einigen Studien - die mechanische Hyperalgesie bei Entzündung praktisch verhindern. Die hyperalgetische Wirkung von NGF kommt über direkte und indirekte Wirkungen am peripheren Neuron zustande. Indirekte Wirkungen sind die Stimulation von Mastzellen und von neutrophilen Granulozyten, sodass diese Serotinin und Histamin freisetzen (beide wirken auf Nozizeptoren), und die Stimulation sympathischer Nervenfasern, sodass diese Prostaglandine freisetzen, die ebenfalls auf die Nozizeptoren wirken. NGF wirkt aber auch direkt auf die Endigungen der Nervenfasern. Die Stimulation der trkA-Rezeptoren erhöht die Empfindlichkeit von Ionenkanälen, die für die Transduktion von Reizen und für die Entstehung von Aktionspotenzialen in der Endigung exprimiert sind (TRPV1-Rezeptoren, ASIC-Ionenkanäle, Natriumkanäle). Des Weiteren wird der durch NGF aktivierte trkA-Rezeptor-Komplex internalisiert und zum Zellkörper transportiert, wodurch die Synthese der Neuropeptide Substanz P, „Calcitonin gene-related peptide“, des TRPV1-Rezeptors und die Synthese eines anderen Wachstumsfaktors („brain derived neurotrophic factor“, BDNF) gefördert wird. Die erhöhte Freisetzung von Substanz P, „Calcitonin generelated peptide" und BDNF im Rückenmark unterstützt die Entstehung der zentralen Sensibilisierung (Übersichten in [2, $4,5])$.

\section{Innovation und Risiko}

Diese Daten verdeutlichen insgesamt die wichtige Rolle von NGF bei der Erhaltung der trkA-exprimierenden Nozizeptoren und bei der entzündungsbedingten Sensibilisierung. NGF ist nicht nur einer von vielen Mediatoren, die Nozizeptoren sensibilisieren, sondern er reguliert zahlreiche Funktionsmoleküle in diesen Nervenzellen. Es liegt daher nahe, NGF mit dem Ziel der Antinozizeption zu neutralisieren. Die berichteten analgetischen Wirkungen in den klinischen Studien bestätigen die in der Grundlagenforschung gezeigte antihyperalgetische Wirkung voll und ganz und lassen es möglich erscheinen, dass mit dieser Therapie der Schmerz „an der Wurzel gepackt“ wird. Andererseits zeigt das Anhalten der Studien von Pfizer, dass im Rahmen dieser Therapie Effekte auftreten können, deren Bedeutung vor einer Fortsetzung der Studien aufgeklärt werden muss.

Für den Verfasser dieses Editorials stellt sich wegen der Schlüsselstellung von NGF insbesondere die Frage, ob man mit der Neutralisierung von NGF möglicherweise zu viel des Guten bewirkt, d. h. die Nozizeption zu sehr reduziert. Um auf den Titel des Editorials zurückzukommen:

Wir brauchen dringend Innovationen in der Schmerztherapie, um insbesondere chronische Schmerzen des Bewegungsapparates besser bekämpfen zu können.

Andererseits birgt der „Zugriff“ auf die Nozizeptoren möglicherweise auch die Gefahr, zu weit zu gehen. Es wird, wie schon angedeutet, sowohl für die Klinik als auch für die Schmerzforschung sehr interessant sein zu sehen, was aus der Vision einer Anti-NGF-Therapie wird.

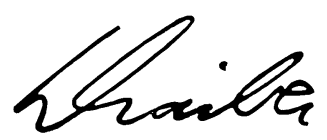

Hans-Georg Schaible

\section{Korrespondenzadresse \\ Prof. Dr. H.-G. Schaible}

Physiologie I, Institut für Physiologie

der Universität Jena

Am Teichgraben 8, 07743 Jena

hans-georg.schaible@mti.uni-jena.de
Interessenkonflikt. Der korrespondierende Autor gibt an, dass kein Interessenkonflikt besteht.

\section{Literatur}

1. Baerwald C (2008) Schmerztherapie bei Arthrose. Schmerztherapie 4:15-17

2. Bennett D (2007) NGF, sensitization of nociceptors. In: Schmidt RF, Willis WD (Hrsg) Encyclopedia of Pain, Volume 2. Springer, Berlin Heidelberg New York Tokyo, S 1338-1342

3. Breivik H, Collett B, Ventafridda V et al (2006) Survey of chronic pain in Europe: prevalence, impact on daily life, and treatment. Eur J Pain 10:287-333

4. McMahon SB, Bennett DLH, Bevan S (2006) Inflammatory mediators and modulators of pain. In: McMahon SB, Koltzenburg M (Hrsg) Wall and Melzack's textbook of pain, 5. Aufl. Elsevier, Churchill Livingstone, S49-72

5. Stein C, Clark JD, Oh U et al (2009) Peripheral mechanisms of pain and analgesia. Brain Res Rev 60:90-113 